\title{
Optical Crosstalk in Fiber-Radio WDM Networks
}

\author{
David Castleford, Student Member, IEEE, Ampalavanapillai Nirmalathas, Member, IEEE, \\ Dalma Novak, Member, IEEE, and Rodney S. Tucker, Fellow, IEEE
}

\begin{abstract}
This paper investigates the impact of optical crosstalk in fiber-radio systems incorporating wavelength division multiplexing. (WDM). We present a simple analytical model that allows the impact of optical crosstalk in such networks to be assessed and validate the results via experiment for both in-band and out-of-band optical crosstalk. We show that crosstalk-induced power penalties in fiber-radio WDM networks are reduced compared to baseband modulation for the case of in-band crosstalk. In addition, in contrast to baseband modulated optical links, the crosstalk channel in fiber-radio systems can be filtered electrically if the crosstalk signal carries a different wireless frequency. However, a power penalty is still observed for the case of in-band crosstalk, even for perfect electrical filtering of the crosstalk channel.
\end{abstract}

Index Terms-Fiber-radio, optical crosstalk, wavelength division multiplexing.

\section{INTRODUCTION}

$\mathbf{F}$ UTURE wireless access networks will be required to deliver a wide variety of services to fixed and mobile users [1]. The limited available electrical spectrum at microwave frequencies and the expected large data rates in such networks will drive the use of smaller radio cells, thereby allowing frequency reuse in nonadjacent cells [2]. Such a wireless network architecture will therefore require a large number of antenna base stations (BSs) in order to ensure adequate radio coverage. The use of optical fiber in this radio access network (producing a hybrid "fiber-radio" system) [1]-[3] promises to reduce the network cost, as well as enable wavelength division multiplexing (WDM) to further increase capacity. The application of WDM in fiber-radio networks has many advantages including simplification of the network topology by allocating different wavelengths to individual BSs, enabling easier network and service upgrades and providing simpler network management [4]-[6].

The requirement for numerous BSs in future fiber-radio networks dictates that the BS cost must be minimized. This may be achieved by reducing the BS hardware and moving the wireless network frequency translation and management equipment to the central office (CO). In designing cost-effective BS architectures, low-cost optoelectronic interfaces will be required, driving the use of WDM optical components with less stringent specifications. In any fiber-radio network incorporating WDM, the impact of optical crosstalk must be considered, taking into account both the access network topology and the BS architecture.

Manuscript received January 15, 2001; revised May 17, 2001.

The authors are with the Australian Photonics Cooperative Research Centre, Photonics Research Laboratory, Department of Electrical and Electronic Engineering, The University of Melbourne, Melbourne, Vic. 3010, Australia.

Publisher Item Identifier S 0018-9480(01)08725-7.
Frequency division multiple access (FDMA) is typically used in the wireless domain in fiber-radio networks, allowing a number of users to use multiple RF frequencies, both in the down- and uplink directions. These multiple radio frequencies can be modulated onto a single optical carrier [2]-[4], [7]. In contrast to crosstalk in optical networks employing baseband digital modulation [8], [9], which has been extensively studied in the literature, little research has been carried out for optical links incorporating subcarrier or analog modulation. Moura et al. [10] considered optical crosstalk arising from reflections in an amplitude shift-keyed (ASK) subcarrier modulation scheme and showed that the impact was identical to that for the case of baseband modulation. In addition, we have previously presented a simple analytical model that allows the impact of both in-band and out-of-band optical crosstalk to be analyzed for the case of an optical link employing binary phase-shift keying (BPSK) carrier modulation. We then confirmed the results through experiment [11].

In this paper, we consider the impact of the RF carrier phase on the resulting optical power penalty and explain the important difference between in-band and out-of-band optical crosstalk. We also consider for the first time typical WDM architectures that may be used in future fiber-wireless networks and explain how these determine the impact of optical crosstalk. We show that appropriate electrical frequency assignment can virtually eliminate the impact of optical crosstalk due to adjacent optical channels through electrical filtering. However, this is not the case when the crosstalk optical channel is at the same wavelength as the signal wavelength, even when the electrical channel is filtered in the electrical domain.

\section{Optical Crosstalk IN FibER-RAdio WDM NetworkS}

Fig. 1 shows a typical unidirectional fiber ring architecture that can be used for the delivery of broad-band wireless services [5], [12]. Every ring carries multiple wavelengths, each of which can originate at or be destined for a given BS. In each WDM ring, each optical wavelength can carry multiple radio channels at intermediate, radio frequencies, or even millimeter-wave frequencies [4]. Optical add-drop multiplexers (OADMs) allow the wavelength carrying downlink wireless frequencies to be transmitted to the desired BS. Upstream signal transmission is achieved via the same wavelength by adding it back into the ring using the same OADM. A different band of optical wavelengths could also be used for downstream and upstream traffic, however, this would increase the complexity of the OADM and network management. As shown in Fig. 1, the CO can be linked to the ring either by a bidirectional link or by two separate unidirectional links. Note that individual COs serve several separate 


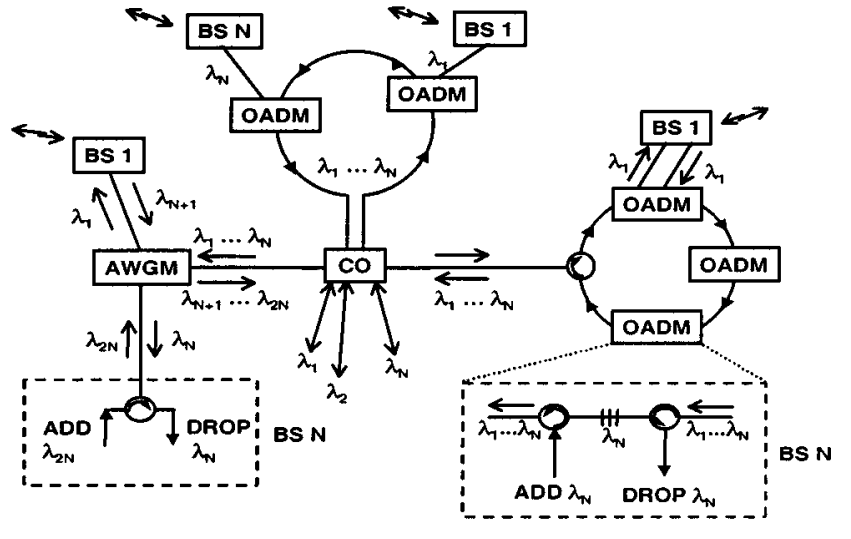

Fig. 1. Fiber-radio WDM network architectures.

WDM rings, enabling both wavelength and wireless frequency reuse. Electrical frequency reuse is also possible within an individual "local" ring.

An alternative WDM fiber-radio network topology is a star topology [4], also shown in Fig. 1. Here, a bi-directional WDM link routes traffic to a single arrayed-waveguide grating multiplexer (AWGM), which distributes different wavelengths to individual BSs. The AWGM allows different add/drop wavelengths to be used at each BS, separated by the free spectral range of the device. This minimizes the OADM complexity and since bi-directional links are used, the use of different upstream and downstream wavelengths will reduce the impact of optical crosstalk compared to the case where the wavelengths are the same. Alternatively, the same wavelengths can be used in both downstream and upstream directions. While two separate unidirectional fibers could be used instead of a single bi-directional fiber, this would double the required number of fibers in the network. In addition, each star could begin directly at the $\mathrm{CO}$, eliminating the need for the AWGM. In the fiber-radio WDM star network topology, each CO addresses several separate stars, making use of both optical wavelength and wireless frequency reuse.

If we consider a fiber-radio network employing FDMA, typically several frequencies are used for each BS, allowing multi-user coverage within the BS cell area. Different frequencies are used in the down- and uplink, thereby minimizing electrical interference. Adjacent BSs are assigned different wireless bands to avoid co-channel interference, while frequency reuse in nonadjacent cells enables the efficient use of the available wireless spectrum. In the WDM optical network architectures shown in Fig. 1, different BSs within a ring or a star are assigned different optical wavelengths. This means that some of the optical wavelengths may carry data at the same wireless frequency (due to electrical frequency reuse). Different rings or stars linked to the same $\mathrm{CO}$ may also reuse the same optical wavelengths, feeding BSs using the same wireless spectrum.

In the network shown in Fig. 1, two types of optical crosstalk can arise. In-band optical crosstalk occurs if the crosstalk signal is at the same wavelength as the desired signal, while out-of-band crosstalk results if the crosstalk is at a different wavelength. In the ring topology depicted in Fig. 1, the OADM comprises two circulators and a fiber Bragg grating (FBG) which is used to reflect the desired BS wavelength back to the first circulator, where it is dropped. However, imperfections in the FBG will result in a fraction of all other wavelengths also being reflected. Such out-of-band crosstalk will depend on the spectral profile of the FBG, however, levels ranging from -25 to $-35 \mathrm{~dB}$ below the desired wavelength optical power are typical [5], [13]. Another possible leakage path is across the circulator linking the ring to the $\mathrm{CO}$, so that upstream channels can be fed back into the downstream path. In the WDM star topology, each AWGM output port in the downstream direction will not only comprise the desired wavelength but also a small fraction of all other channels (also ranging from -25 to -35 $\mathrm{dB}$ [14]). When bi-directional links are used between the $\mathrm{CO}$ and AWGM and between the AWGM and each BS, any source of optical reflections will cause out-of-band crosstalk between the upstream and downstream optical signals. Sources of optical reflections include Rayleigh backscatter in the fiber or reflections due to imperfect or damaged optical components or splices ( -40 to $-10 \mathrm{~dB}$ below the signal optical power).

Unlike out-of-band crosstalk, in-band crosstalk cannot be filtered. For example, in the OADM in Fig. 1, the added wavelength is reflected by the FBG and transmitted back to the CO. However some of the "add" wavelength will leak through the grating and be received at the "drop" port (typically $-30 \mathrm{~dB}$ below the "drop" channel power). Similarly, some of the dropped wavelength will pass through the grating and continue around the ring, together with the added wavelength (also approximately $-30 \mathrm{~dB}$ ). While it is unlikely that in-band optical crosstalk carrying data at the same RF frequency as the signal can occur within an individual ring or star fed by a $\mathrm{CO}$, it may arise between different rings or stars within a local $\mathrm{CO}$.

\section{TheORETICAL MODELS FOR OpticAl CROSSTALK}

In this section, we extend previously used theoretical models [8]-[11] to assess the impact of optical crosstalk in fiber-radio networks. We consider both in-band and out-of-band optical crosstalk as well as two possible scenarios for the crosstalk wireless channel: the implementation of electrical frequency reuse where the crosstalk electrical carrier frequency is the same as the signal carrier frequency, and the use of different wireless frequencies leading to different crosstalk and signal carrier frequencies. A simple model of in-band crosstalk is developed first considering the presence of a signal wavelength and one crosstalk wavelength. An externally modulated optical link is assumed where a modulated subcarrier at frequency $\omega_{R F}$ drives the modulator. The electric field associated with the desired and crosstalk optical signals that are at the same wavelength can then be expressed as

$$
\begin{aligned}
& E_{1}(t)=E_{1} \cos \left(\omega t+\theta_{1}\right)\left[1+m_{1} \alpha(t) \cos \left(\omega_{\mathrm{R} F} t+\phi_{1}\right)\right]^{1 / 2}, \\
& E_{2}(t)=\sqrt{x} E_{1} \cos \left(\omega t+\theta_{2}\right)\left[1+m_{2} \beta(t) \cos \left(\omega_{R F} t+\phi_{2}\right)\right]^{1 / 2}
\end{aligned}
$$

where $E_{1}, E_{2}$ are the electric fields of the optical signals, $\omega$ is the optical frequency, $m_{1}$ and $m_{2}$ are the modulation indexes, $\alpha(t)$ and $\beta(t)$ are the signal and crosstalk data, $x$ is the optical 


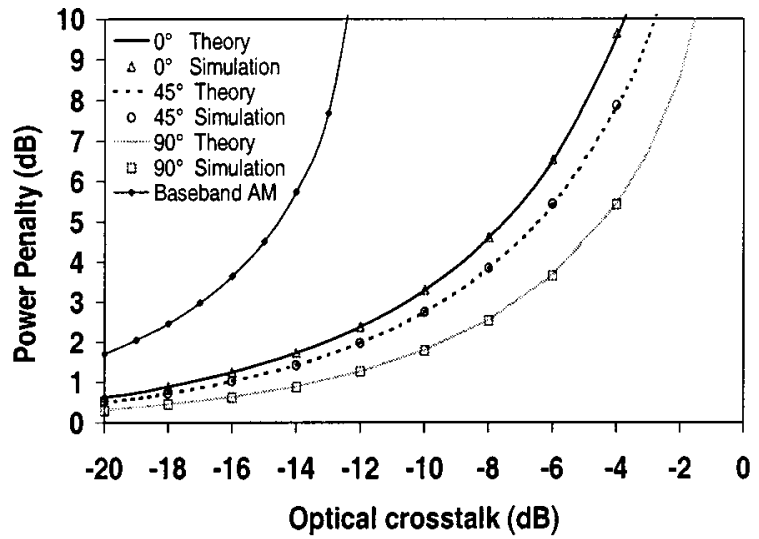

Fig. 2. Theoretical and simulated optical power penalties versus in-band optical crosstalk with frequency reuse as a function of RF carrier phase difference.

crosstalk power ratio, $\theta_{1}$ and $\theta_{2}$ are the optical phase, and $\phi_{1}, \phi_{2}$ are the electrical phases. The optical crosstalk ratio is defined as the ratio of the crosstalk optical power to the signal optical power. After photodetection (and assuming a first-order approximation), a set of frequency components at $\omega_{\mathrm{RF}}$ result [11]. For the case of an RF subcarrier modulated by data in BPSK format, the baseband data is recovered by a phase-locked loop (PLL) which must be locked to the phase of the signal electrical carrier, i.e., $\phi_{1}$. The signal waveform $I_{\text {down }}$ of the downconverted baseband data channel can then be represented by

$$
\begin{aligned}
I_{\text {down }} \propto m_{1} \alpha[1+\sqrt{x} & \cos (\Delta \theta)] \\
& +m_{2} \beta \sqrt{x} \cos (\Delta \phi)[\sqrt{x}+\cos (\Delta \theta)]
\end{aligned}
$$

where $\Delta \phi=\phi_{1}-\phi_{2}$ is the RF phase difference between the signal channel and crosstalk signal, and $\Delta \theta=\theta_{1}-\theta_{2}$ is the optical phase difference between the two optical carriers. Equation (2) shows that, due to the multiplication effect of the PLL, the mixing products arising from the crosstalk signal itself and intermixing terms due to the signal carrier and crosstalk sidebands beating, are multiplied by $\cos (\Delta \phi)$. This RF phase difference is clearly important since orthogonal RF carriers are rejected by the PLL. Equations (1) and (2) can then be used to evaluate bit error rates (BERs) as a function of total optical power for varying crosstalk levels, allowing optical power penalties at a BER of $10^{-9}$ to be calculated for BPSK modulation [11].

Fig. 2 shows the optical power penalty predicted using the analytical model for in-band incoherent crosstalk, plotted as a function of optical crosstalk level for RF phase differences of $\Delta \phi=0^{\circ}, 45^{\circ}$, and $90^{\circ}$. For comparison, the predicted power penalties for baseband data transmission are also shown using the same analytical approach, however, for baseband amplitude modulation with a decision threshold equal to half the average total power [8]. Fig. 2 shows that the optical power penalties arising from in-band optical crosstalk depend on the RF phase difference between the crosstalk and signal carriers. In addition, the power penalty is significantly worse for baseband data transmission. It is also apparent that a large penalty is still present for the case of $90^{\circ} \mathrm{RF}$ phase difference, even though no crosstalk electrical signal is present at the output of the receiver. This is caused by the presence of the crosstalk optical carrier, which

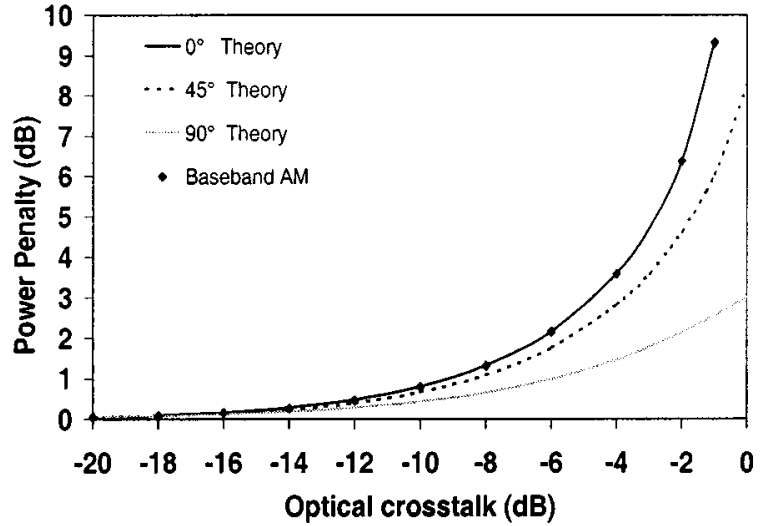

Fig. 3. Theoretical optical power penalties as a function of out-of-band crosstalk with frequency reuse use as a function of RF carrier phase difference.

beats with the signal data sidebands thereby varying the resulting signal data amplitude. Since we consider RF carrier frequencies and data rates which are larger than the optical carrier linewidth, we assume all results are independent of optical linewidth. In order to confirm the analytical model, a commercially available optical link design simulation tool was used to analyze the effect of in-band crosstalk ${ }^{1}$ and these simulation results are also presented in Fig. 2. Very close agreement with the analytical model was obtained.

We now consider optical crosstalk arising from an optical signal at a different wavelength from the desired channel but modulated by the same RF frequency due to frequency reuse. Following the previous analysis, the expression for the downconverted signal is

$$
I_{\text {down }} \propto m_{1} \alpha(t)+x m_{2} \beta(t) \cos (\Delta \phi) .
$$

A comparison of (2) and (3) shows that out-of-band crosstalk is only influenced by the RF phase difference, and therefore a reduction of crosstalk is possible when $\Delta \phi=90^{\circ}$. In addition, the signal and crosstalk data are recovered separately and no mixing terms between the signal and crosstalk are present at the RF frequency. This then results in so-called linear or additive crosstalk. Fig. 3 shows the predictions of the analytical model for out-of-band crosstalk for $\Delta \phi=0^{\circ}, 45^{\circ}$ and $90^{\circ}$, as a function of optical crosstalk ratio. Also shown in the diagram is the predicted power penalty for baseband transmission. The actual power penalty at a particular crosstalk ratio is less for out-of-band crosstalk than for the in-band case. Fig. 3 shows that, for an RF phase difference of $\Delta \phi=90^{\circ}$, when the crosstalk signal is at the same optical power as the desired channel (i.e., $0 \mathrm{~dB}$ of out-of-band optical crosstalk), the optical power penalty is $3 \mathrm{~dB}$ (the total power has doubled). This is as expected since the $90^{\circ}$ phase shift leads to a cancellation of the crosstalk contribution.

In contrast to in-band crosstalk where the optical power penalty for baseband data transmission is significantly higher than for RF subcarrier transmission (at all crosstalk ratios), Fig. 3 shows that out-of-band crosstalk-induced power penalties for baseband data transmission are identical to those for

\footnotetext{
${ }^{1}$ Virtual Photonics Inc., [Online]. Available: http://www.virtualphotonics.com
} 
RF subcarrier transmission when the carriers are in-phase. This is expected, as the crosstalk is linear irrespective of the modulation format or receiver structure.

If we consider a crosstalk signal at the same wavelength as the signal channel but carrying data at a different wireless carrier frequency, the results are different. Although the wireless frequencies carried by the desired wavelength and crosstalk signal are presumably sufficiently separated so that electrical filtering can minimize the effect of adjacent electrical channels, in the optical domain, the optical carriers overlap (for in-band crosstalk). Mixing terms are produced in the photodetector, although the crosstalk sidebands beating with the signal and crosstalk carrier now produce a signal at a different RF frequency. However, even if we assume perfect filtering, the presence of the crosstalk optical carrier still ensures that the signal amplitude depends on the crosstalk ratio and optical phase difference, as shown below:

$$
I_{\text {down }} \propto m_{1} \alpha(t)[1+\sqrt{x} \cos (\Delta \theta)] .
$$

The amplitude of the desired signal comprises an extra term due to beating between the crosstalk optical carrier and signal optical sidebands. This situation is in effect the same as that for crosstalk at the same RF carrier frequency but with an RF phase difference of $90^{\circ}$, since recovery of the wireless frequency also leads to elimination of the crosstalk electrical signal in this situation, as shown in Fig. 2.

As discussed earlier, out-of-band crosstalk results in a received electrical signal that is a sum of the electrical signals carried by each individual wavelength. If the crosstalk wavelength carries data at different electrical frequencies to the signal wavelength, then these can be filtered electrically, ensuring minimal impact on the signal quality. Hence, the level of electrical crosstalk can be determined purely by the electrical channel separation and channel and filter bandwidths [15]. It should also be remembered that $-10 \mathrm{~dB}$ of optical crosstalk corresponds to a $-20-\mathrm{dB}$ level of electrical crosstalk, so that optical component specifications may be sufficient to ensure an adequate electrical carrier-to-noise ratio without the need for additional electrical filtering. Furthermore, electrical filtering of adjacent channels within the transmitted signal wireless band may be enough to ensure out-of-band optical crosstalk has a minimal effect.

\section{EXPERIMENT}

This section describes the experiments used to verify the theoretical model for out-of-band and in-band optical crosstalk with and without frequency reuse. Fig. 4 shows the experimental setup for measurement of out-of-band optical crosstalk. Two separate optical paths implement the desired channel and crosstalk signal, each comprising a distributed feedback (DFB) laser $\left(\lambda_{1}=1549.4 \mathrm{~nm}, \lambda_{2}=1550 \mathrm{~nm}\right)$ and an electrooptic modulator (EOM). Separate data channels at $155 \mathrm{Mb} / \mathrm{s}$ modulate an RF signal at $2 \mathrm{GHz}$ in BPSK modulation format. An RF phase shifter was used to control the RF phase difference between the signal channel and crosstalk signal. The two optical signals are coupled together and amplified using an erbium-doped fiber amplifier (EDFA) with a gain of $23 \mathrm{~dB}$ in order to compensate for losses in the optical link. The input

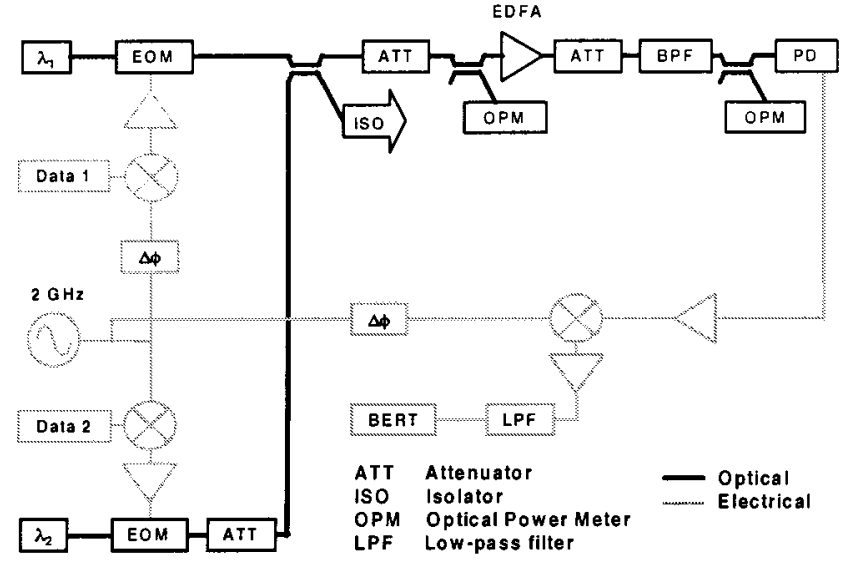

Fig. 4. Experimental setup for measurement of out-of-band optical crosstalk.

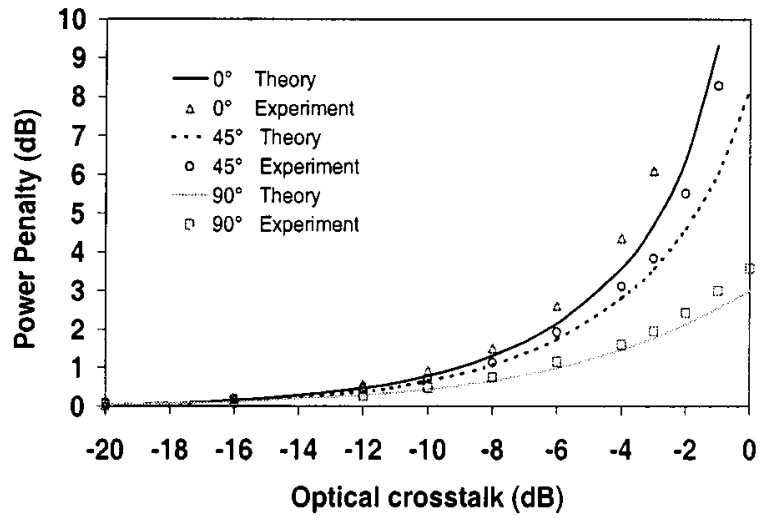

Fig. 5. Measured and predicted optical power penalties versus out-of-band crosstalk with frequency reuse as a function of RF carrier phase difference.

power to the EDFA is kept constant during the experiment while the crosstalk level is varied. An optical bandpass filter (BPF) with a bandwidth of $2 \mathrm{~nm}$ was included after the EDFA in order to filter out excess amplified spontaneous emission noise. At the receiver, the optical signals are detected using a $\mathrm{p}$-i-n photodetector (PD) and the same 2-GHz signal is used to downconvert the detected modulated RF signals and recover the data. Electrical amplifiers are used in the receiver together with a $150-\mathrm{MHz}$ low-pass filter. Polarization controllers are used to ensure signal and crosstalk are polarization-aligned at the photodetector. A BER testset (BERT) measures the BER of the recovered $155-\mathrm{Mb} / \mathrm{s}$ data as a function of optical crosstalk ratio (varied via the optical attenuator in the optical path of $\lambda_{2}$ ). A BER of $10^{-9}$ is obtained for a received optical input power at the PD input of $-19 \mathrm{dBm}$. Fig. 5 shows the measured optical power penalty at a BER of $10^{-9}$ as a function of optical crosstalk ratio, for $\Delta \phi=0,45^{\circ}$ and $90^{\circ}$, plotted alongside the theoretical predictions for out-of-band crosstalk. The measurements show very good agreement with theory, with a small discrepancy between the two sets of results appearing at higher crosstalk levels $(>-4 \mathrm{~dB})$.

The setup used to measure in-band crosstalk is very similar to that shown in Fig. 4; however, here a single DFB laser at wavelength $\lambda_{1}$ provides both the desired optical signal and the in-band crosstalk component. A 2-km-long spool of single-mode fiber (SMF) is included in the crosstalk path so 


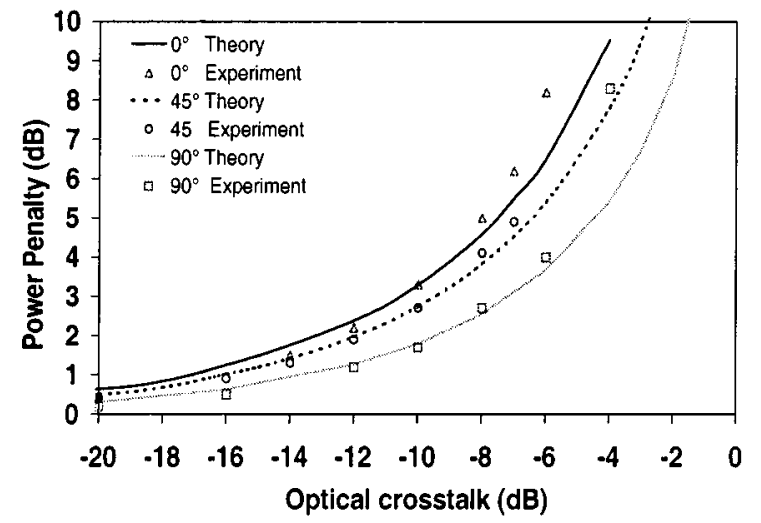

Fig. 6. Measured and predicted optical power penalties versus in-band crosstalk with frequency reuse as a function of RF carrier phase difference.

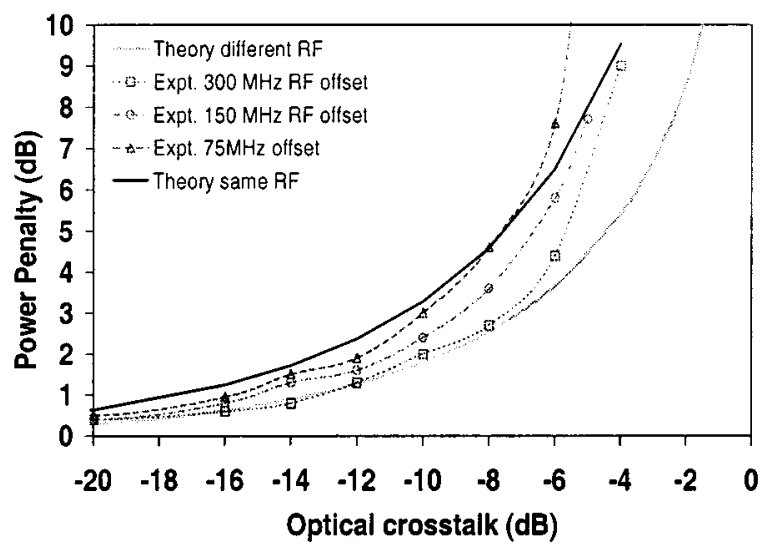

Fig. 7. Measured and predicted optical power penalties due to in-band optical crosstalk with different electrical frequencies.

that the optical signals are rendered incoherent. The two optical signals are each externally modulated by an RF carrier at $3.5 \mathrm{GHz}$ carrying $155-\mathrm{Mb} / \mathrm{s}$ data in BPSK modulation format (two separate data generators provide the $155-\mathrm{Mb} / \mathrm{s}$ radio data). Any harmonics of the $3.5-\mathrm{GHz}$ carrier were attenuated by the 4.2-GHz bandwidth of the electrical amplifier. Fig. 6 shows the measured optical power penalties due to in-band optical crosstalk in a fiber-radio network with radio frequency reuse (where the optical channel and crosstalk signals carry the same wireless frequency at $3.5 \mathrm{GHz}$ ). Also shown for comparison are the predicted power penalties calculated for the possible situation of an RF phase difference between the desired and crosstalk signals, of $0^{\circ}, 45^{\circ}$, and $90^{\circ}$.

The experimental setup in Fig. 4 was also used to investigate the impact of in-band crosstalk as a function of the frequency separation between the wireless signal and crosstalk carrier frequency. A separate RF signal source was used for the crosstalk RF signal, allowing the crosstalk frequency to be varied. Fig. 7 shows the measured power penalties for in-band crosstalk where the crosstalk carrier frequency is offset by 75 , 150, and $300 \mathrm{MHz}$. Also shown are theoretical predictions for the case of perfect electrical filtering of the crosstalk carrier, which is the best case, and for identical RF frequencies that are in-phase, which is the worst case. The importance of the electrical frequency separation is evident showing the importance of electrical filtering. If we consider a power penalty of $1 \mathrm{~dB}$, the allowed maximum crosstalk varies from -13 to $-17 \mathrm{~dB}$ depending on whether the signal and crosstalk carrier frequencies are identical or different (and filtered).

\section{DISCUSSION}

The previous sections have considered the effect of both in-band and out-of-band crosstalk in WDM fiber-radio systems, taking into account the situation where a crosstalk channel may carry data at different frequencies. The results show that optical crosstalk may not necessarily impose serious constraints on optical component specifications, even for the case of in-band crosstalk. This means that wavelength reuse at each BS is possible in a ring architecture for example, as it is more spectrally efficient than using different wavelengths in the upand downstream. However, the optical component crosstalk level may not be the only factor determining the optical power penalty. If we consider, for example, crosstalk arising at a BS between downstream and upstream wavelengths, the downstream wavelength optical power may be lower than the upstream wavelength, changing the effective crosstalk level. Since we are considering fiber-radio systems with limited radio bandwidth, frequency reuse is essential in a real wireless network. While different wireless frequencies will be used in the down- and uplink, for the case of in-band crosstalk arising at the BS this does not eliminate the effect of optical crosstalk as a power penalty is still observed. In contrast, if we consider out-of-band crosstalk, using different frequency bands for adjacent optical wavelengths clearly allows the impact of crosstalk to be virtually eliminated, since the only effect of the crosstalk channels is to increase the total optical power (assuming perfect electrical filtering).

The importance of RF carrier phase difference was highlighted earlier for the case of frequency reuse. For BPSK modulation an orthogonal crosstalk carrier can significantly relax crosstalk requirements or resulting power penalties. Unfortunately, it is unlikely that this situation can be guaranteed or controlled in the fiber-radio network itself. For the case of in-band crosstalk, the crosstalk channel will either be a delayed version of the original signal or originate from a different part of the network, so that the actual electrical phase difference between the signal and crosstalk carriers will depend on path length. For out-of-band crosstalk, the carrier phase difference at the receiver will depend on the fiber path length and chromatic dispersion even though the same RF generator may have been used at the $\mathrm{CO}$ during modulation. Both in-band and out-of-band crosstalk may also experience polarization mode dispersion, resulting in a slowly time-varying phase difference [16]. The various RF oscillators used in the network may also drift slowly over time relative to one other, resulting in a similar effect. Hence worst-case carrier phase alignment should be assumed when assessing the expected optical power penalties.

\section{CONCLUSION}

We have presented a theoretical and experimental investigation of the impact of in-band and out-of-band optical crosstalk in fiber-radio systems incorporating WDM. It was shown that the $\mathrm{RF}$ carrier phase of the crosstalk wireless signal is important in 
determining penalties and that for BPSK subcarrier modulation the impact of in-band crosstalk is reduced compared to baseband modulation. Minimal crosstalk will also occur if the crosstalk wireless frequency can be filtered in the electrical domain, although a power penalty still exists for the case of in-band optical crosstalk. An important conclusion from this work is that relaxation of the specifications of WDM components in fiber-radio WDM systems may be achieved provided appropriate optical wavelength and electrical frequency planning is implemented.

\section{ACKNOWLEDGMENT}

The authors are grateful to Dr. S. Dods for useful discussions.

\section{REFERENCES}

[1] H. Ogawa, D. Polifko, and S. Banba, "Millimeter-wave fiber optics systems for personal radio communication," IEEE Trans. Microwave Theory Tech., vol. 40, pp. 2285-2293, Dec. 1992.

[2] T. S. Chu and M. J. Gans, "Fiber optic microcellular radio," IEEE Trans. Veh. Technol., vol. 40, pp. 599-606, Aug. 1991

[3] A. J. Cooper, "Fiber/radio' for the provision of cordless/mobile telephony services in the access network," Electron. Lett., vol. 26, pp. 2054-2056, 1990.

[4] G. H. Smith, D. Novak, and C. Lim, "A millimeter-wave full-duplex fiber-radio star-tree architecture incorporating WDM and SCM," IEEE Photon. Technol. Lett., vol. 10, pp. 1650-1652, Nov. 1998.

[5] R. Heinzelmann, T. Kuri, K. Kitayama, A. Stöhr, and D. Jäger, "Optical add-drop multiplexing of $60 \mathrm{GHz}$ millimeterwave signals in a WDM radio-on-fiber ring," in Proc. OFC, Baltimore, MD, 2000, paper FH4-1.

[6] R. A. Griffin, P. M. Lane, and J. J. O'Reilly, "Radio-over-fiber distribution using an optical millimeter-wave/DWDM overlay," in Proc. OFC, vol. 2, USA, 1999, pp. 70-72

[7] R. Olshansky, V. A. Lanzisera, and P. M. Hill, "Subcarrier multiplexed lightwave systems for broad-band distribution," J. Lightwave Technol., vol. 7, pp. 1329-1342, Sept. 1989.

[8] E. L. Goldstein, L. Eskildsen, and A. F. Elrefaie, "Performance implications of component crosstalk in transparent lightwave networks," IEEE Photon. Technol. Lett., vol. 6, pp. 657-660, May 1995.

[9] P. J. Legg, M. Tur, and I. Andonovic, "Solution paths to limit interferometric noise induced performance degradation in ASK/direct detection lightwave networks," J. Lightwave Technol., vol. 14, pp. 1943-1954, Sept. 1996.

[10] L. Moura, M. Darby, P. M. Lane, and J. J. O'Reilly, "Impact of interferometric noise on the remote delivery of optically generated millimeter-wave signals," IEEE Trans. Microwave Theory Tech., vol. 45 , pp. 1398-1402, Aug. 1997.

[11] D. Castleford, A. Nirmalathas, and D. Novak, "Impact of optical crosstalk in fiber-radio systems incorporating WDM," in Proc. Int. Top. Meet. Micro. Photon., Oxford, U.K., 2000, paper TU1.6.

[12] H. Kim and Y. C. Chung, "Bi-directional passive optical network for CDMA personal communication service," in Proc. OFC, vol. 2, 1999, Paper WD5, pp. 67-69.

[13] C. Yang and Y. Lai, "Apodised fiber Bragg gratings fabricated with uniform phase mask using low cost apparatus," Electron. Lett., vol. 36, pp. 655-657, 2000

[14] H. Yamada, K. Takada, Y. Inoue, K. Okamoto, and S. Mitachi, "Low-crosstalk arrayed-waveguide grating multi/demultiplexer with phase compensating plate," Electron. Lett., vol. 33, pp. 1698-1699, 1997.

[15] R. D. Feldman, T. H. Wood, and R. F. Austin, "Operation of a frequency shift keyed subcarrier multiple-access system for a passive optical network in the presence of strong adjacent channel interference," IEEE Photon. Technol. Lett., vol. 7, pp. 427-430, Apr. 1995.

[16] C. D. Poole, R. W. Tkach, A. R. Chraplyvy, and D. A. Fishman, "Fading in lightwave systems due to polarization-mode dispersion," IEEE Photon. Technol. Lett., vol. 3, pp. 68-70, Jan. 1991.

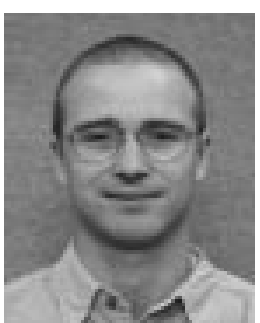

David Castleford ( $\left.\mathrm{S}^{\prime} 00\right)$ received the B.Sc. degree in physics (with honors) from the University of Manchester, Manchester, U.K., in 1993, the M.Sc. degree in the physics of laser communications from the University of Essex, Essex, U.K., in 1995, and is currently working toward the Ph.D. degree at the Photonics Research Laboratory at The University of Melbourne, Melbourne, Australia.

From 1996 to 1999, he was with Alcatel Submarine Networks, London, U.K., where he was involved with the branching unit and repeater design and development. His current work is on wavelength division multiplexing fiber-radio systems and optical crosstalk.

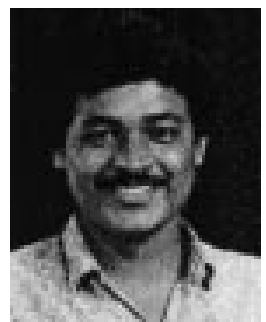

Ampalavanapillai Nirmalathas (M'97) received the B.E. (with honors) and $\mathrm{Ph}$.D. degrees in electrical and electronic engineering from The University of Melbourne, Melbourne, Australia, in 1993 and 1997. respectively.

In 1997, he joined the Photonics Research Laboratory (PRL) (a member of the Australian Photonics CRC), The University of Melbourne, where he is currently a Senior Lecturer. He is also the Research Director of the PRL and Manager of the Telecommunications Technologies Research Program in the Australian Photonics CRC. His research interests include fiber-optic feed networks for wireless systems, modeling of optical and wireless communication systems, high-speed communication technologies, and optical networks.

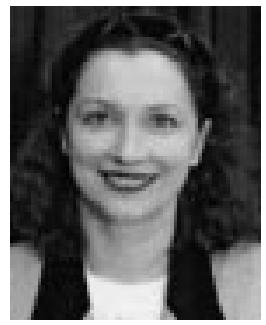

Dalma Novak (S'90-M'91) received the Bachelor of Engineering (electrical) (with first-class honors) and the Ph.D. degree from the University of Queensland, Brisbane, Australia, in 1987 and 1992, respectively.

In 1992, she joined the PRL, Department of Electrical and Electronic Engineering, The University of Melbourne, Melbourne, Australia, where she is currently an Associate Professor and Reader. She is also a Key Researcher in the CRC and Deputy Director of the PRL. She has authored or co-authored over 120 papers in these areas. Her research interests include fiber-wireless communication systems, semiconductor lasers, and high-speed optical networks.

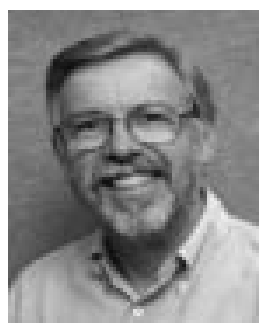

Rodney S. Tucker (S'72-M'75-SM'85-F'90) is Director of the ARC Special Research Centre for Ultra-Broadband Information Networks at The University of Melbourne, Melbourne, Australia. $\mathrm{He}$ is also associated with the Australian Photonics Cooperative Research Centre. From 1973 to 1975, he was a Lecturer in Electrical Engineering at the University of Melbourne. During 1975 and 1976, he was a Harkness Fellow with the Department of Electrical Engineering and Computer Sciences, University of California at Berkeley. From 1976 to 1977, he was a Harkness Fellow with the School of Electrical Engineering, Cornell University, Ithaca, NY. From 1977 to 1978, he was with Plessey Research (Caswell) Ltd., Caswell, U.K.. From 1978 to 1983, he was with the Department of Electrical Engineering, University of Queensland, Brisbane, Australia. From 1984 to 1990, he was with the Crawford Hill Laboratory, AT\&T Bell Laboratories, Holmdel, NJ. In 1990, he joined the Department of Electrical and Electronic Engineering, The University of Melbourne.

Dr. Tucker is a Fellow of the Australian Academy of Technological Sciences and Engineering and the Australian Academy of Science. He was an editor of the IEEE TRANSACTIONS ON MICROWAVE THEORY AND TECHNIQUES. He is currently an associate editor of the IEEE PHOTONICS TECHNOLOGY LETTERS. In 1997, he was awarded the Australia Prize for his contributions to telecommunications. 


\section{University Library}

\section{- M M I E E R VA A gateway to Melbourne's research publications}

Minerva Access is the Institutional Repository of The University of Melbourne

Author/s:

Castleford, D;Nirmalathas, A;Novak, D;Tucker, RS

Title:

Optical crosstalk in fiber-radio WDM networks

Date:

2001-10-01

Citation:

Castleford, D., Nirmalathas, A., Novak, D. \& Tucker, R. S. (2001). Optical crosstalk in fiberradio WDM networks. IEEE TRANSACTIONS ON MICROWAVE THEORY AND TECHNIQUES, 49 (10), pp.2030-2035. https://doi.org/10.1109/22.954826.

Publication Status:

Published

Persistent Link:

http://hdl.handle.net/11343/33777 